[Article]

www.whxb.pku.edu.cn

\title{
钯铁合金催化剂的稳定性
}

\author{
柯 曦 ${ }^{1}$ 崔国峰 ${ }^{2}$ 沈培康 ${ }^{1, *}$ \\ ('中山大学物理科学与工程技术学院, 光电材料与技术国家重点实验室, 广州 510275 ; \\ 2 中山大学化学与化学工程学院, 广州 510275 )
}

\begin{abstract}
摘要：分别在酸性和碱性溶液中, 结合旋转圆盘电极技术和电化学石英晶体微天平技术原位考察了钯铁合金 催化剂对氧还原反应催化活性的稳定性. 发现在酸性溶液中, 钯铁合金催化剂对氧还原反应的催化活性不稳定, 原因是钯铁合金催化剂在酸性溶液中发生电化学/化学溶解; 在碱性溶液中, 覆盖在电极表面的钯铁合金催化剂 的质量及电化学活性面积在电化学扫描过程中不发生明显变化, 保持对氧还原反应的催化活性, 证明钯铁合金 催化剂在碱性介质中非常稳定.
\end{abstract}

关键词：钯铁合金; 电化学石英晶体微天平; 催化剂; 燃料电池; 稳定性 中图分类号: $0643 ; 0646$

\section{Stability of Pd-Fe Alloy Catalysts}

\author{
$\mathrm{KE} \mathrm{Xi}^{1} \quad$ CUI Guo-Feng ${ }^{2} \quad$ SHEN Pei-Kang ${ }^{1, *}$ \\ ('State Key Laboratory of Optoelectronic Materials and Technologies, School of Physics and Engineering, \\ Sun Yat-Sen University, Guangzhou 510275, P. R. China; ${ }^{2}$ School of Chemistry and Chemical Engineering, \\ Sun Yat-Sen University, Guangzhou 510275, P. R. China)
}

\begin{abstract}
The stability of Pd-Fe alloy catalysts for the oxygen reduction reaction (ORR) was characterized in situ by using the rotating disk electrode and electrochemical quartz crystal microbalance (EQCM) techniques, both in acidic and alkaline solutions. The alloy catalyst was found to be unstable in the acidic solution because of the electrochemical and chemical dissolution of the catalyst. This dissolution resulted in a reduction of activity for the oxygen reduction reaction and also a reduction in the mass of the catalyst. In an alkaline solution, the mass of the Pd-Fe alloy catalyst deposited on the electrode surface and its electrochemically active surface area hardly changed. Consequently, the catalyst kept its activity for stable oxygen reduction.
\end{abstract}

Key Words : Pd-Fe alloy; Electrochemical quartz crystal microbalance; Catalyst; Fuel cell; Stability

质子交换膜燃料电池(PEMFCs) 至今没有实现 规模化生产的主要原因之一是成本太高, 其中催化 剂占了相当大的比例, 因为目前广泛使用的催化剂 是以铂为主的催化剂. 为降低铂金属的用量, 将其与

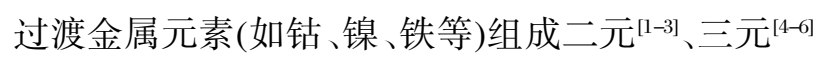
合金催化剂或使用非铂材料 ${ }^{[7,8}$ 受到了广泛的研究. 在阴极氧还原反应催化剂中, Shao 等踯报道了钯铁 合金催化剂具有很强的氧还原催化活性, 当钯铁原
子比为 3:1 时, 其氧还原催化活性甚至要强于铂. 然 而, 这些非铂合金催化剂的稳定性的研究还相当有 限 ${ }^{[9-11]}$.

本研究的目的是结合旋转圆盘电极技术和电化 学石英晶体微天平技术, 分别在酸性溶液 $(0.1 \mathrm{~mol}$ $\mathrm{L}^{-1}$ 硫酸)和碱性溶液 $\left(0.1 \mathrm{~mol} \cdot \mathrm{L}^{-1}\right.$ 氢氧化钾 $)$ 中, 对钯 铁合金催化剂的稳定性进行原位研究. 由于具有原位 检测电极/溶液界面达到纳克级的质量变化, 电化学

\footnotetext{
Received: August 28, 2008; Revised: October 28, 2008; Published on Web: December 4, 2008.

*Corresponding author. Email: stsspk@mail.sysu.edu.cn; Tel: +8620-84036736.
}

广东省科技基金(2007A010700001, 2007B090400032)和广州市科技基金(2007Z1-D0051, SKT[2007]17-11)资助项目

(C) Editorial office of Acta Physico-Chimica Sinica 
石英晶体微天平已经被广泛应用于电化学各个领域 的研究中, 如电沉积 ${ }^{[12]}$ 、离子/有机小分子的吸附 ${ }^{[13,14]}$ 、 欠电位沉积 ${ }^{[15]}$ 、腐蚀过程研究 ${ }^{[16]}$. 根据 Sauerbrey 方 程 ${ }^{[17]}$ :

$$
\Delta m=-\frac{A \sqrt{\mu_{\mathrm{q}} \rho_{\mathrm{q}}}}{2 f_{0}^{2}} \Delta f=-C_{\mathrm{f}} \Delta f
$$

其中, $\Delta f$ 为频率变化值, $\Delta m$ 为发生在电极/溶液界 面的法拉第过程中的质量变化, $f_{0}$ 为晶体谐振基频, $\mu_{\mathrm{q}}$ 为石英晶体的剪切模量, $\rho_{\mathrm{q}}$ 为石英晶体的密度, $A$ 为石英晶体具有压电活性的几何面积, $C_{\mathrm{f}}$ 为标定常 数. 晶体共振频率的变化量与电极表面的质量变化 量具有确定的线性关系, 因而根据所测得的频率变 化就可以获得电化学过程中的质量变化信息, 为理 解电化学反应机理提供数据.

\section{1 实 验}

\section{1 试剂与装置}

$\mathrm{H}_{2} \mathrm{SO}_{4}(99 \%) 、 \mathrm{KOH}(82 \%) 、 \mathrm{PdCl}_{2}(59.5 \%) 、 \mathrm{~K}_{2} \mathrm{SO}_{4}$ $(99 \%)$ 和 $\mathrm{FeSO}_{4} \cdot 7 \mathrm{H}_{2} \mathrm{O}(99 \%)$ 均为分析纯, 所有溶液均 用二次去离子水配制 (电阻率为 $18 \mathrm{M} \Omega$ ). 电化学石 英晶体微天平实验在三电极体系电解池中进行, 通 过石英晶体微天平 (QCM 922, Prinston Applied Research, 美国)和恒电位仪(Potentiostat 2273, Prinston Applied Research, 美国) 联合控制. 在旋转圆盘电极 实验中, 玻碳电极用作研究电极基底; 在电化学石 英晶体微天平实验中, 基频为 $9 \mathrm{MHz}$ 的镀金AT-切石 英晶体电极 (镀金薄膜电极的几何面积为 $0.196 \mathrm{~cm}^{2}$ ) 用作工作电极, 铂片 $(1.0 \mathrm{~cm} \times 1.0 \mathrm{~cm})$ 用作对电极, 饱 和甘永电极 (SCE, 酸性溶液中) 和永/氧化永电极 $(\mathrm{Hg} / \mathrm{HgO}$, 碱性溶液中) 用作参比电极.

\section{2 实验程序}

碳载钯铁合金催化剂 $(\mathrm{PdFe} / \mathrm{C})$ 的制备方法与参 考文献[7]相同. 在旋转圆盘电极实验中, 工作电极 的准备过程如下: 取 $5 \mathrm{mg}$ 钯铁合金催化剂粉末分散 在 $0.9 \mathrm{~mL}$ 醇和 $0.1 \mathrm{~mL}$ Nafion (5\% Nafion, DuPont, 美国)的混合溶液中, 超声分散 $15 \mathrm{~min}$, 用微量进样 器取 $15 \mu \mathrm{L}$ 涂覆在玻碳电极上, 干燥以备用. 在电 化学石英晶体微天平实验中, 首先将石英晶体电极 用 50/50(体积比)的 $30 \%$ 双氧水和浓硫酸混合溶液 清洗约 $20 \mathrm{~s}$, 然后用大量去离子水清洗电极. 石英晶 体微天平电极标定常数 $C_{\mathrm{f}}$ 以及电极表面粗糙度 $R$ 的确定过程如下: 根据 Vatankhah 等 ${ }^{[18]}$ 的建议, 通过 测试 $3 \mathrm{mmol} \cdot \mathrm{L}^{-1}$ 硝酸银溶液中银离子在镀金石英
晶体电极上的电沉积, 测得 $C_{\mathrm{f}}$ 的值约为 $1.1 \mathrm{ng} \cdot \mathrm{Hz}^{-1}$, 这与根据 Sauerbrey 方程计算得到的理论值 1.093 $\mathrm{ng} \cdot \mathrm{Hz}^{-1}$ 非常接近. 根据文献[19]推荐的氧吸附方法 测得镀金石英晶体电极的粗鋉度为 1.3. 钯铁合金采 取电沉积的方法制备: 电镀液为 $5 \mathrm{mmol} \cdot \mathrm{L}^{-1} \mathrm{PdCl}_{2}+$ $10 \mathrm{mmol} \cdot \mathrm{L}^{-1} \mathrm{FeSO}_{4} \cdot 7 \mathrm{H}_{2} \mathrm{O}+0.1 \mathrm{~mol} \cdot \mathrm{L}^{-1} \mathrm{~K}_{2} \mathrm{SO}_{4}$, 采取 恒电位的方法沉积, 具体细节可以参考文献[20,21]. 所沉积合金的体相分析采用配有能谱的扫描电镜 (SEM-EDS, Quanta 400F, 法国)进行分析, 测得所沉 积的合金成分含有钯和铁, 且以钯为主. 根据沉积前 后晶体共振基频的变化, 可以计算出所沉积的合金 的质量约为 $1 \mu \mathrm{g}$, 将沉积了合金的电极用去离子水 清洗, 然后在 $0.1 \mathrm{~mol} \cdot \mathrm{L}^{-1}$ 高氯酸中用循环伏安法扫 描, 以获得稳定的曲线. 在每次测试前, 溶液都要用 高纯氮除氧 $20 \mathrm{~min}$, 在实验时, 在液面上吹氮气以保 持惰性气氛. 所有实验均在室温下进行(约 $25^{\circ} \mathrm{C}$ ).

\section{2 结果与讨论}

\section{1 钯铁合金催化剂的旋转圆盘电极研究}

图 1(a)为涂覆在玻碳电极上的碳载钯铁合金催 化剂在酸性溶液中的初始及经过 300 和 600 圈循环 扫描后的循环伏安曲线. 从图中可以看出, 随着扫描 次数的增多, 催化剂的电化学活性面积急剧减小, 从 而导致催化剂对氧还原过程的催化性能的降低(图 $1(b))$.

图 2(a)为碳载钯铁合金催化剂在碱性溶液中的 初始及经过 540 圈循环扫描后的循环伏安曲线. 从 位于- $0.2 \mathrm{~V}(\mathrm{vs} \mathrm{Hg} / \mathrm{HgO})$ 左右的电极表面氧化物还原 峰的面积来看, 虽然催化剂的电化学活性面积随着 扫描次数的增多而略有减小, 但减小的程度不大. 相 应的氧还原实验(图 2(b)) 表明, 随着循环扫描次数 的增加, 起始还原电压略有负移, 极限电流略有减 少. 总体来讲, 在碱性溶液中, 催化剂电化学活性面 积与对氧还原过程催化能力并不随电位循环扫描次 数的增加而发生显著变化, 显示了较好的稳定性.

\section{2 钯铁合金的电化学石英晶体微天平研究}

图 3 为镀有钯铁合金的石英晶体电极在 0.1 $\mathrm{mol} \cdot \mathrm{L}^{-1}$ 硫酸溶液中的循环伏安曲线和相应的质量 变化曲线. 从图中可以看出, 在循环扫描一圈后, 质 量变化曲线并不闭合, 而是减少了, 说明电极表面合 金在扫描过程中发生电化学/化学溶解, 与文献[22] 中的结果一致. 经过循环扫描一圈后电极表面合金 的质量减少约 $90 \mathrm{ng}$. 合金的溶解速率(定义为每循 

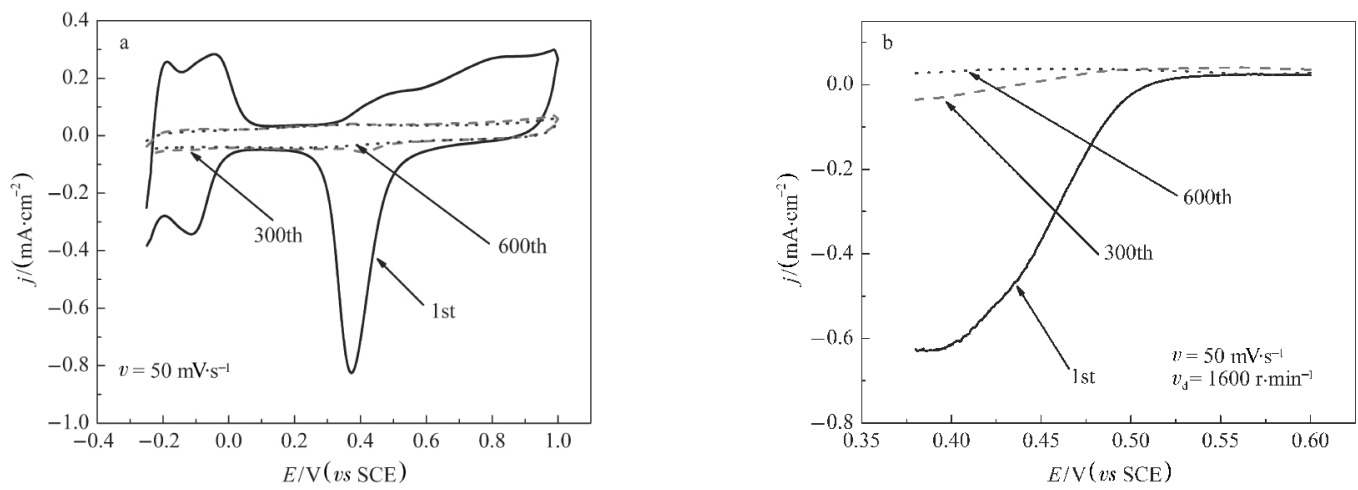

图 1 (a) PdFe/C 在 $0.1 \mathrm{~mol} \cdot \mathrm{L}^{-1} \mathrm{H}_{2} \mathrm{SO}_{4}$ 溶液中的循环伏安曲线和(b)在氧饱和的 $0.1 \mathrm{~mol} \cdot \mathrm{L}^{-1} \mathrm{H}_{2} \mathrm{SO}_{4}$ 溶液中相应的 氧还原反应曲线

Fig.1 (a) Cyclic voltammograms of $\mathrm{PdFe} / \mathrm{C}$ in $0.1 \mathrm{~mol} \cdot \mathrm{L}^{-1} \mathrm{H}_{2} \mathrm{SO}_{4}$ solution and (b) the corresponding oxygen reduction reaction (ORR) performance in oxygen saturated $0.1 \mathrm{~mol} \cdot \mathrm{L}^{-1} \mathrm{H}_{2} \mathrm{SO}_{4}$ solution
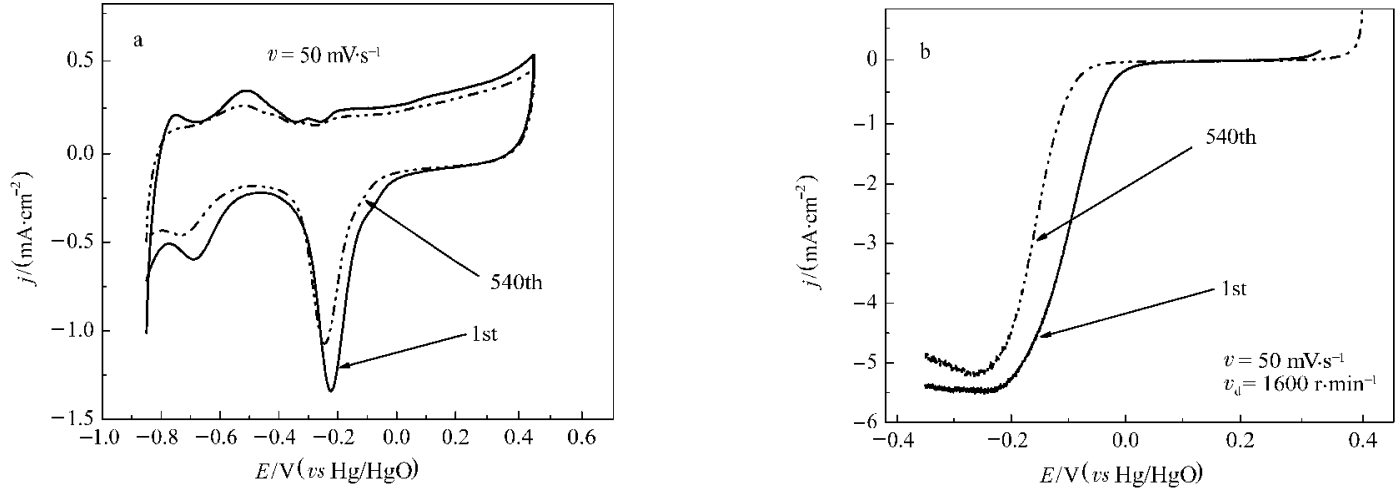

图 2 (a) $\mathrm{PdFe} / \mathrm{C}$ 在 $0.1 \mathrm{~mol} \cdot \mathrm{L}^{-1} \mathrm{KOH}$ 溶液中的循环伏安曲线和(b)在氧饱和的 $0.1 \mathrm{~mol} \cdot \mathrm{L}^{-1} \mathrm{KOH}$ 溶液中相应的 氧还原反应曲线

Fig.2 (a) Cyclic voltammograms of $\mathrm{PdFe} / \mathrm{C}$ in $0.1 \mathrm{~mol} \cdot \mathrm{L}^{-1} \mathrm{KOH}$ solution and (b) the corresponding ORR performance in oxygen saturated $0.1 \mathrm{~mol} \cdot \mathrm{L}^{-1} \mathrm{KOH}$ solution

环扫描一圈后电极表面合金质量的减少量)与电位 扫描上限有关. 如图 4 所示, 合金的溶解速率随电

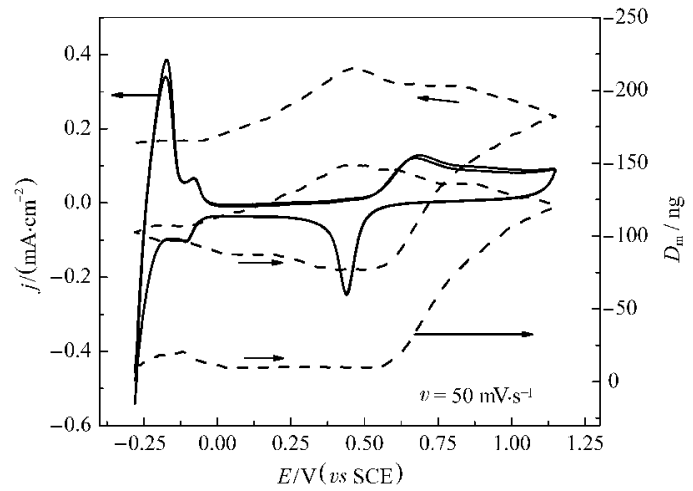

图 3 钯铁覆盖的 $\mathrm{EQCM}$ 电极在 $0.1 \mathrm{~mol} \cdot \mathrm{L}^{-1} \mathrm{H}_{2} \mathrm{SO}_{4}$ 溶液 中的循环伏安曲线(实线)和相应的质量变化曲线(虚线)

Fig.3 Cyclic voltammograms of Pd-Fe covered

EQCM electrode in $0.1 \mathrm{~mol} \cdot \mathrm{L}^{-1} \mathrm{H}_{2} \mathrm{SO}_{4}$ solution (solid line) and the corresponding EQCM response (dashed line)
位扫描上限 $\left(E_{\mathrm{a}}\right)$ 的变化而变化. 当电位扫描上限小于 $0.6 \mathrm{~V}$ 时, 合金几乎不发生溶解; 当电位上限大于 0.6

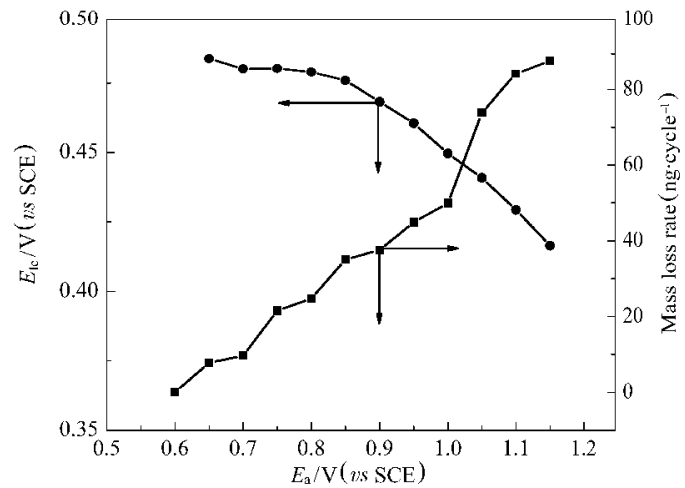

图 4 表面氧还原极限电流电位 $\left(\boldsymbol{E}_{\mathrm{lc}}\right)$ 、电极表面合金溶解速 率与扫描电位上限 $\left(\boldsymbol{E}_{\mathrm{a}}\right)$ 的关系

Fig.4 Relationship between the potential at limiting current $\left(E_{\mathrm{lc}}\right)$, mass loss rate and anodic potential $\left(E_{\mathrm{a}}\right)$ 

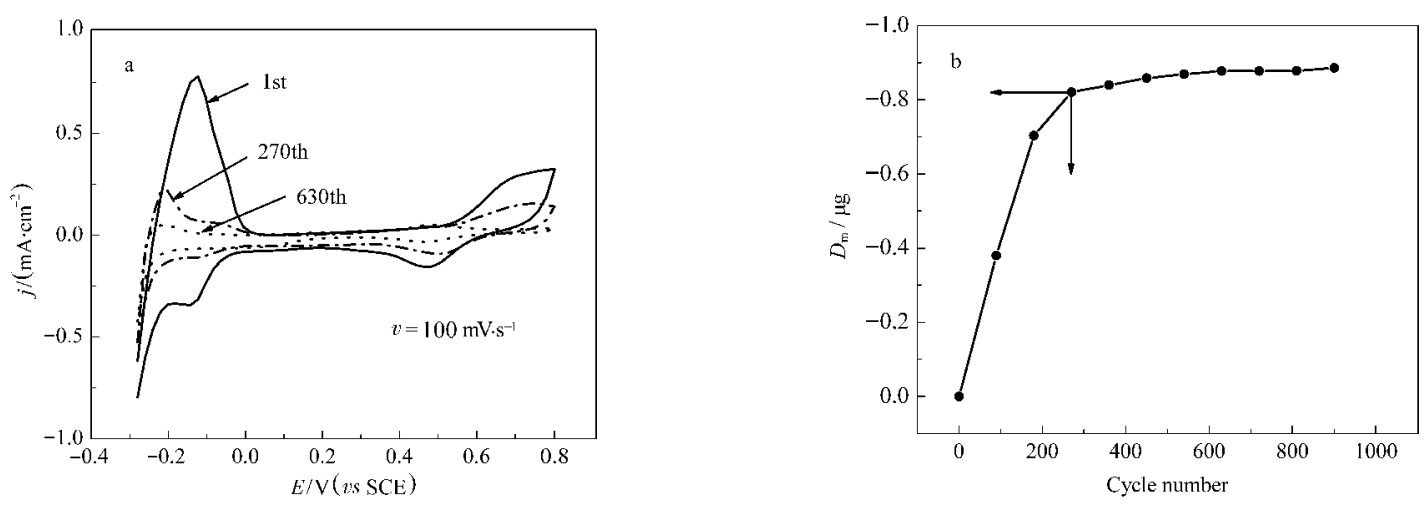

图 5 (a) 钯铁覆盖的 $\mathrm{EQCM}$ 电极在 $0.1 \mathrm{~mol} \cdot \mathrm{L}^{-1} \mathrm{H}_{2} \mathrm{SO}_{4}$ 溶液中的循环伏安曲线及(b) 电极表面合金溶解量与扫描圈数的关系

Fig.5 (a) Cyclic voltammograms of Pd-Fe covered EQCM electrode in $0.1 \mathrm{~mol} \cdot \mathrm{L}^{-1} \mathrm{H}_{2} \mathrm{SO}_{4}$ solution and (b) the relationship between the total mass loss and cycle number

$\mathrm{V}$ 时, 合金在扫描过程中发生溶解. 溶解速率随着 $E_{\mathrm{a}}$ 的增大而增大, 当电位上限达到 $1.15 \mathrm{~V}$ 时, 溶解 速率达到约 $90 \mathrm{ng} \cdot \mathrm{cycle}^{-1}$. 在负向扫描过程中, 表面 氧还原极限电流电位 $\left(E_{\mathrm{lc}}\right)$ 与电位扫描上限的关系如 图 4 所示. 从图中可以看出, 还原极限电流电位随扫 描上限的增大而逐渐负移. 这是由于电位扫描上限 越大, 正向扫描过程中所形成的氧化物薄膜越厚, 导 致还原极限电流电位负移.

图 5 为镀有钯铁合金的石英晶体电极在 0.1 $\mathrm{mol} \cdot \mathrm{L}^{-1}$ 硫酸溶液中的稳定性测试结果. 从图 $5(\mathrm{a})$ 可 以看到, 循环扫描导致催化剂对氢的吸脱附峰面积 逐渐减小, 即催化剂的电化学活性面积不断减小. 图 5(b)所示为电极表面合金溶解量与循环扫描圈数的 关系. 从图中可以看出, 随着扫描的进行, 电极表面 合金不断溶解, 在扫描了 270 圈以后, 所沉积的钯铁 合金的绝大部分已溶解, 质量的变化趋于零.

图 6 为在 $0.1 \mathrm{~mol} \cdot \mathrm{L}^{-1}$ 氢氧化钾溶液中的电化 学石英晶体微天平测试结果. 图 6(a)为碱性溶液中

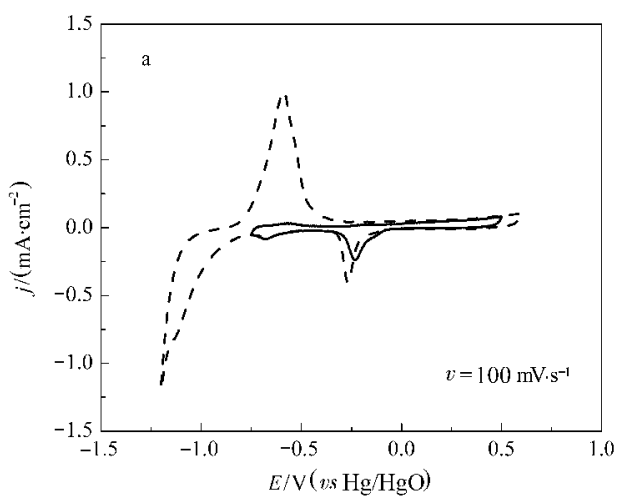

钯铁合金覆盖石英晶体电极在不同电位区间扫描的 循环伏安曲线. 图 6(b)为相对应的频率变化曲线. 虚 线对应的电位扫描区间为从氢吸脱附区到氧吸脱附 区, 实线对应的电位扫描区间为从双层充放电区到 氧吸脱附区. 从图中(图 6(b), 虚线)可以看出, 当电 位负向扫描至氢吸附区时, 氢的吸附使晶体共振频 率减小, 从而导致频率变化曲线不闭合. 当电位扫描 区间不包含氢吸脱附区间时, 在经历电位循环扫描 后, 频率变化曲线是闭合的(图 6(b), 实线). 即在碱 性溶液中, 循环扫描并不导致电极表面合金的显著 溶解. 频率变化曲线的闭合也说明了在氧还原反应 中, 催化剂的活性是稳定的.

图 7 为钯铁覆盖的石英晶体电极在 $0.1 \mathrm{~mol} \cdot \mathrm{L}^{-1}$ 氢氧化钾溶液中的稳定性测试结果. 从图 7(a)可以 看出, 在经历电位循环扫描后, 循环伏安曲线并未发 生明显变化. 即电极表面合金的电化学活性面积并 未随着扫描的进行而急剧变化. 有趣的是, 从图 7(b) 可见, 在扫描过程中, 晶体共振频率变小, 而根据图

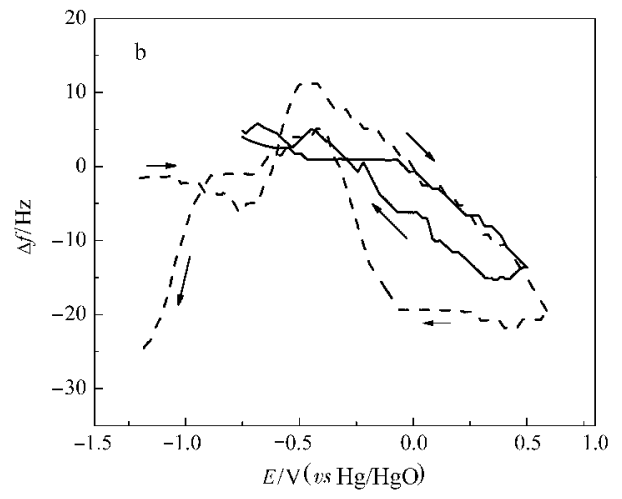

图 6 (a) 钯铁覆盖的 $\mathrm{EQCM}$ 电极在 $0.1 \mathrm{~mol} \cdot \mathrm{L}^{-1} \mathrm{KOH}$ 溶液中的循环伏安曲线和 $(\mathrm{b})$ 相应的频率变化曲线

Fig.6 (a) Cyclic voltammograms of Pd-Fe covered EQCM electrode in $0.1 \mathrm{~mol} \cdot \mathrm{L}^{-1} \mathrm{KOH}$ solution and (b) the corresponding frequency change curve 

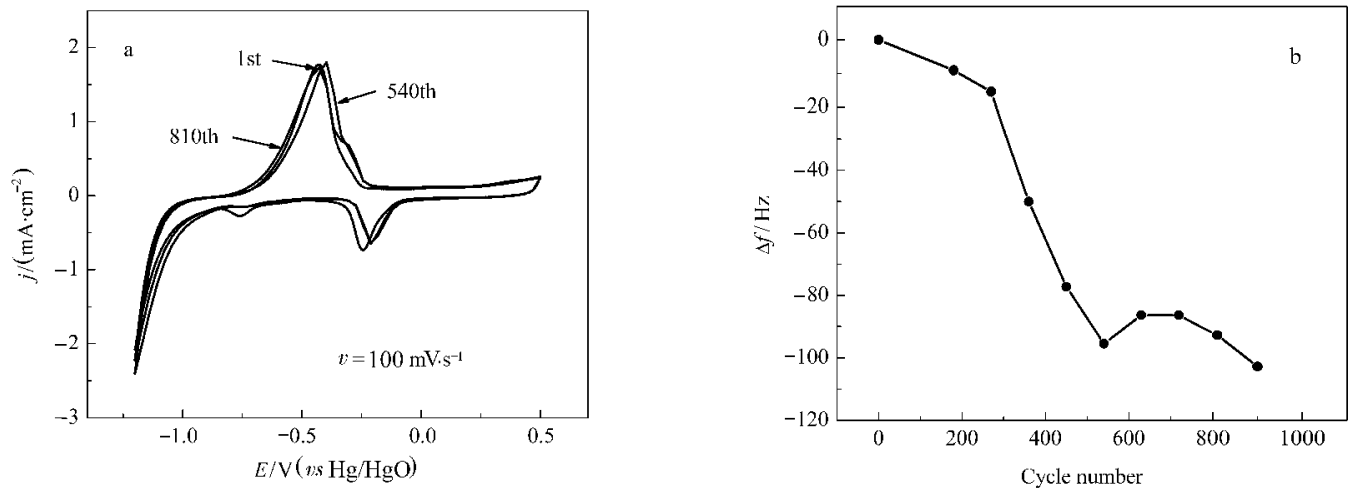

图 7 (a) 钯铁覆盖的 $\mathrm{EQCM}$ 电极在 $0.1 \mathrm{~mol} \cdot \mathrm{L}^{-1} \mathrm{KOH}$ 溶液中的循环伏安曲线和 $(\mathrm{b})$ 相应的频率变化与扫描圈数的关系

Fig.7 (a) Cyclic voltammograms of Pd-Fe covered EQCM electrode in $0.1 \mathrm{~mol} \cdot \mathrm{L}^{-1} \mathrm{KOH}$ solution and (b) relationship between the frequency change and cycle number

7(a)可知, 催化剂电化学活性面积的变化很小, 因而 表面合金质量变化很小. 这说明导致晶体共振频率 不断减小的因素并不是电极表面合金质量的变化. 对这种现象可能的解释是: 由于钯具有吸氢储氢的 能力, 在长时间的电位扫描过程中, 吸附的氢可能未 得到完全释放, 因而在晶体内部产生应力, 导致晶体 共振频率变小 ${ }^{[23,24]}$.

\section{3 结 论}

结合旋转圆盘电极技术和电化学石英晶体微天 平技术, 分别在酸性和碱性溶液中原位考察了钯铁 合金催化剂的稳定性. 在酸性溶液中, 由于钯铁合金 的电化学/化学溶解, 导致催化剂的电化学活性面积 不断减小, 对氧还原反应的催化性能逐渐降低, 因而 钯铁合金催化剂在酸性溶液中不稳定; 在碱性溶液 中, 钯铁合金催化剂的电化学活性面积并不随电化 学反应的进行发生明显变化, 对氧还原反应的催化 性能并不发生明显变化, 表明钯铁合金催化剂在碱 性溶液中非常稳定.

\section{References}

1 Yano, H.; Kataoka, M.; Yamashita, H.; Uchida, H.; Watanabe, M. Langmuir, 2007, 23: 6438

2 Mani, P.; Srivastava, R.; Strasser, P. J. Phys. Chem. C, 2008, 112 2770

3 Antolini, E.; Salgado, J. R. C.; Gonzalez, E. R. J. Electroanal. Chem., 2005, 580: 145

4 Riberio, J.; dos Anjos, D. M.; Kokoh, K. B.; Coutanceau, C.; Leger, J. M.; Olivi, P.; de Andrade, A. R.; Tremiliosi-Filho, G. Electrochim. Acta, 2007, 52: 6997

5 Colmati, F.; Antolini, E.; Gonzalez, E. R. J. Alloys Compounds, 2008, 456: 264
6 Whitacre, J. F.; Valdez, T. I.; Narayanan, S. R. Electrochim. Acta, 2008, 53: 3680

7 Song, S. Q.; Wang, Y.; Tsiakaras, P.; Shen, P. K. Appl. Catal. B, 2008, 78: 381

8 Shao, M. H.; Sasaki, K.; Adzic, R. R. J. Am. Chem. Soc., 2006, 128: 3526

9 Feng, Y. J.; Alonso-Vante, N. Physica Status Solidi B, 2008, 245 1792

10 Kim, J. H.; Ishihara, A.; Mitsushima, S.; Kamiya, N.; Ota, K. I. Electrochim. Acta, 2007, 52: 2492

11 Sarkar, A.; Murugan, A. V.; Manthiram, A. J. Phys. Chem. C, 2008, 112: 12037

12 Martin, A. J.; Chaparro, A. M.; Daza, L. J. Power Sources, 2007, 169: 65

13 Santos, M. C.; Miwa, D. W.; Machado, S. A. S. Electrochem. Commun., 2000, 2: 692

14 Gonzalez-Pena, O. I.; Chapman, T. W.; Vong, Y. M.; AntanoLopez, R. Electrochim. Acta, 2008, 53: 5549

15 Nicic, I.; Liang, J.; Cammarata, V.; Alanyalioglu, M.; Demir, U.; Shannon, C. J. Phys. Chem. B, 2002, 106: 12247

16 Hepel, M.; Cateforis, E. Electrochim. Acta, 2001, 46: 3801

17 Sauerbrey, G. Z. Phys., 1959, 155: 206

18 Vatankhah, G.; Lessard, J.; Jerkiewicz, G.; Zolfaghari, A.; Conway, B. E. Electrochim. Acta, 2003, 48: 1613

19 Trasatti, S.; Petrii, O. A. J. Electroanal. Chem., 1992, 327: 353

20 Grden, M.; Czerwinski, A. J. Solid State Electrochem., 2008, 12 375

21 Grden, M.; Kumierczyk, K.; Czerwinski, A. J. Solid State Electrochem., 2002, 7: 43

22 Grden, M.; Kotowski, J.; Czerwinski, A. J. Solid State Electrochem., 1999, 3: 348

23 Liu, S. Y.; Kao, Y. H.; Su, Y. O.; Perng, T. P. J. Alloys Compounds, 2001, 316: 280

24 Lukaszewski, M.; Czerwinski, A. J. Electroanal. Chem., 2006, 589: 87 